\section{Revista Chilena \\ de Pediatría}

www.revistachilenadepediatria.cl



www.scielo.cl

\title{
Recomendación para la sospecha, manejo y seguimiento del compromiso cardiovascular en pacientes con Síndrome Inflamatorio Multisistémico en Pediatría asociado a COVID-19 (PIMS-TC). Declaración de Posición de la Sociedad Chilena de Pediatría (SOCHIPE) y Sociedad Chilena de Cardiología y Cirugía Cardiovascular (SOCHICAR)
}

Recommendation for the recognition, management and follow up of cardiovascular compromise in patients with Pediatric Multisystemic Inflammatory Syndrome associated with COVID-19 (PIMS-CT). Position statement of Chilean Scientific Societies

Patricia Álvarez Z. a,b, Guillermo Larios G. ${ }^{c}$, Lida Toro R. ${ }^{\mathrm{d}}$, Valeria Acevedo A. ${ }^{\mathrm{a}, \mathrm{b}}$, Francisca Arancibia G. ${ }^{c}$, Luis Cárdenas M. ${ }^{\text {ef,g, }}$, Carlos Fernández C. ${ }^{\text {hi,j }}$

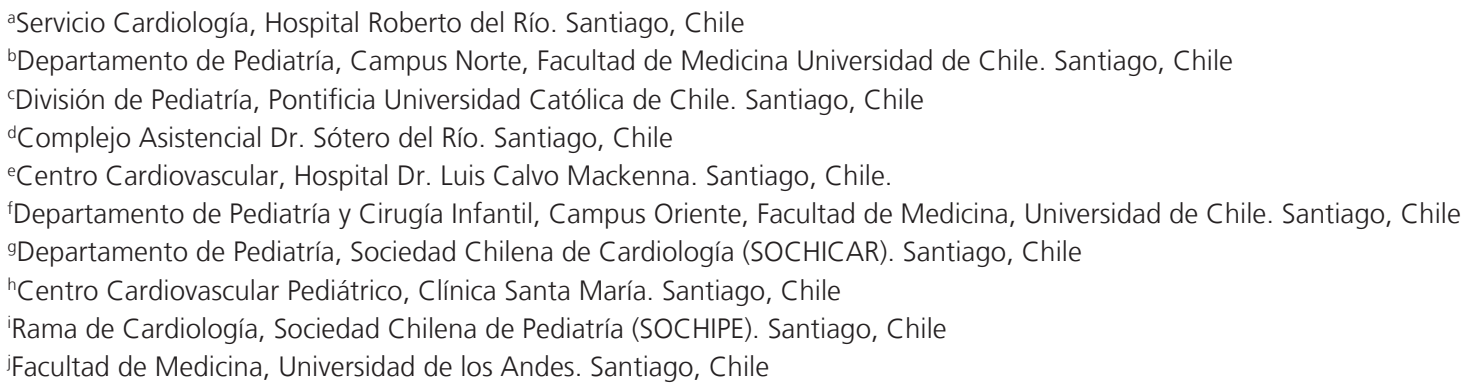

Recibido: 19 de agosto de 2020; Aceptado: 20 de agosto de 2020

¿Qué se sabe del tema que trata este estudio?

El Síndrome Inflamatorio Multisistémico en Pediatría asociado a SARS-CoV-2 (PIMS-TC) cursa con frecuencia con compromiso cardiovascular. Su presencia se asocia a una evolución mas grave, requerimiento de hospitalizaciones en cuidados intensivos y manejo con terapia inmunomoduladora y antiinflamatoria.
¿Qué aporta este estudio a lo ya conocido?

El presente articulo aporta recomendaciones dirigidas a los medicos involucrados en la atención de los pacientes con compromiso cardiovascular en el contexto de PIMS-TC, con el objetivo de orientar la sospecha diagnóstica, su manejo intrahospitalario y el seguimiento ambulatorio.

Correspondencia:

Carlos Fernández C.

carlosfernandez72@yahoo.com 


\section{Resumen}

Desde la aparición de la pandemia por SARS-CoV-2, la población pediátrica ha sido menos afectada por la enfermedad tanto en frecuencia como en severidad. Sin embargo, desde abril de este año se han reportado casos de presentación y gravedad variables, caracterizados por fenómenos inflamatorios que afectan múltiples órganos, condición denominada Síndrome Inflamatorio Multisistémico Pediátrico (PIMS). La literatura describe frecuente compromiso cardíaco, hasta en un $80 \%$. Este se caracteriza por injuria miocárdica con significativa elevación de biomarcadores: Troponinas séricas I/T, BNP o NT-ProBNP, unido a diversos grados de disfunción ventricular, pericarditis, valvulitis y arritmias. Además, se ha evidenciado la presencia de compromiso coronario el cual puede ocurrir hasta en un $23 \%$ de los casos, en un rango que va desde dilataciones hasta aneurismas. El seguimiento cardiológico hospitalizado y ambulatorio se ha sistematizado en base a los fenotipos clínicos de presentación: injuria miocárdica (miocarditis, valvulitis, pericarditis), shock (habitualmente de tipo "vasopléjico"), manifestaciones tipo Enfermedad de Kawasaki y aquellos casos PIMS que no cumplen con la clínica de los tres precedentes. Este último grupo es el que representa el mayor desafío en el corto, mediano y seguimiento a largo plazo. Por esta razón se requiere un equipo multidisciplinario para su manejo. Considerando la alta frecuencia del compromiso cardíaco en el PIMS y la importancia de lograr un consenso en su manejo y seguimiento, se presentan estas recomendaciones según el estado actual del conocimiento de esta patología recientemente descrita.

\section{Abstract}

Since the onset of the SARS-CoV-2 pandemic, the pediatric population has been less affected by the disease both in frequency and severity. However, since April cases of variable presentation and severity characterized by inflammatory phenomena that affect multiple organs have been reported, a condition called Multisystem Inflammatory Syndrome in Children (MIS-C). The literature has reported frequent cardiac involvement, up to $80 \%$. This is characterized by myocardial injury with a significant increase of biomarkers such as serum troponins I and T, BNP, or NT-ProBNP coupled with varying degrees of ventricular dysfunction, pericarditis, valvulitis, and arrhythmias. Coronary compromise has also been described, which can occur in up to $23 \%$ of cases, and ranges from dilatations to aneurysms. Inpatient and outpatient cardiology follow-up has been systematized based on the clinical phenotypes such as myocardial injury (myocarditis, valvulitis, pericarditis), shock (usually vasoplegic), Kawasaki disease-type manifestations, and those MIS-C that do not comply with the clinic of the previous three. This last group represents the main challenge in the short-, medium- and long-term follow-up, therefore, it is necessary a multidisciplinary team for managing these patients. Considering the high frequency of cardiac compromise in MIS-C, and the importance of reaching a consensus regarding its management and follow-up, we present these recommendations according to the current state of knowledge regarding this recently described pathology.
Palabras clave: Síndrome Inflamatorio Multisistémico; PIMS; COVID-19; Injuria Miocárdica; Aneurisma Coronario; SARS-CoV-2; Enfermedad de Kawasaki

Keywords:

Multisystem

Inflammatory

Syndrome;

MIS-C;

COVID-19;

Myocardial Injury;

Coronary Aneurysms;

SARS-CoV-2;

Kawasaki Disease

\section{Introducción}

Desde la declaración de la pandemia por SARSCoV-2 en marzo de 2020 por la OMS, la afección en la edad pediátrica ha sido menos frecuente y de un curso clínico mas leve que en la población adulta, excepto en los menores de un año en los cuales existe una mayor severidad por patología respiratoria. A pesar de este menor compromiso, desde mediados de abril de este año en países de Europa y posteriormente Estados Unidos, se ha reportado un nuevo síndrome inflamatorio multisistémico en niños de expresividad clínica variable, asociado temporalmente con la exposición a SARS-CoV-2, con un desfase variable de 3 a 6 semanas ${ }^{1}$. Este cuadro muestra algunos rasgos clínicos y de laboratorio similares a los observados en la enfermedad de Kawasaki (EK), el síndrome de shock tóxico (SST) o síndrome de activación macrofágica (SAM), lo que motivó a diferentes instituciones a publicar recomendaciones para su sospecha y diagnóstico ${ }^{2,3,4}$. Los estudios han descrito que puede evolucionar de forma grave, con shock, miocarditis y en ocasiones dilatación $\mathrm{y}$ aneurismas coronarios, requiriendo tratamiento en las unidades de cuidados intensivos pediátricos. Esta nueva enfermedad, denominada PIMS-TC (de su sigla en inglés Pediatric Multisystem Inflammatory Syndrome Temporally Associated with SARS-CoV-2) se caracteriza por síntomas gastrointestinales y compromiso miocárdico en una elevada frecuencia ${ }^{5,6}$. Los fenotipos clínicos reportados son heterogéneos y en 
algunos afectados pueden superponerse o adicionarse. Para efecto de esta recomendación se describirán cuatro formas clínicas:

1. Pacientes con signos y síntomas similares a la enfermedad de Kawasaki (EK) o símilar a ésta (EK "like") con o sin compromiso coronario,

2. Presentación clínica de shock ya sea cardiogénico o distributivo.

3. Pacientes con elementos clínicos o de laboratorio sugerentes de formas aisladas o en combinación con: injuria miocárdica, miocarditis, pericarditis, valvulitis y arritmia.

4. Pacientes con signos clínicos y de laboratorio de inflamación, sin elementos que permitan clasificarlos en los tres grupos precedentes (los más frecuentes de acuerdo a las series reportadas actualmente $)^{7}$.

En todas estas formas de presentación se ha encontrado algún elemento de compromiso cardiológico ${ }^{7}$. Esto resulta particularmente importante en el último grupo de pacientes pues constituye un desafío a considerar en cuanto a seguimiento a corto, mediano y largo plazo.

Por lo expuesto, y debido a la frecuencia y magnitud del compromiso cardiovascular, es que cardiólogos de distintos centros del país hemos generado este documento para orientar la sospecha y manejo de estos pacientes.

\section{Compromiso Cardiovascular}

El compromiso cardíaco ha sido descrito hasta en un $80 \%$ de los pacientes y puede producir: derrame pericárdico, miocarditis, valvulitis, compromiso coronario y arritmias ${ }^{5}$ cuyo espectro de manifestación comprende desde formas subclínicas hasta aquellas con requeririmiento de apoyo vasoactivo e inotrópico e incluso, asistencia ventricular extracorpórea (ECMO). Las diferentes frecuencias de aparición descritas en la literatura se describen en la tabla 1.

Tabla 1. Principales frecuencias de Manifestaciones de Compromiso cardiovascular reportadas en la literatura ${ }^{5,7,19,20,21}$

\begin{tabular}{|c|c|c|c|c|}
\hline \multirow[b]{2}{*}{ Tipo compromiso } & \multirow[b]{2}{*}{ \% Total } & \multicolumn{3}{|c|}{ Edad (años) } \\
\hline & & $<6$ & $6-13$ & $13-20$ \\
\hline Cualquier compromiso CV & $80 \%$ & & & \\
\hline Dolor torácico & $11 \%$ & $3 \%$ & $7 \%$ & $27 \%$ \\
\hline Taquicardia & $97 \%$ & $90 \%$ & $100 \%$ & $100 \%$ \\
\hline Hipotensión & $32 \%$ & $10 \%$ & $40 \%$ & $46 \%$ \\
\hline Shock & & $13 \%$ & $12 \%$ & $4 \%$ \\
\hline Uso de DVA & $48-62 \%$ & $48 \%$ & $69 \%$ & $65 \%$ \\
\hline Uso de VM & & $10 \%$ & $7 \%$ & $15 \%$ \\
\hline ECMO & $4 \%$ & $3 \%$ & $5 \%$ & $4 \%$ \\
\hline \multicolumn{5}{|l|}{ Elevación de biomarcadores } \\
\hline BNP $(>400)$ o NT-ProBNP & $73-90 \%$ & $96 \%$ & $100 \%$ & $72 \%$ \\
\hline Troponina US & $50-71 \%$ & $58 \%$ & $81 \%$ & $84 \%$ \\
\hline $\begin{array}{l}\text { Disfunción ventricular } \\
\text { Leve o moderada (FEVI 45-55\%) } \\
\text { Severa (FEVI <30\%) }\end{array}$ & $\begin{array}{c}38-72 \% \\
33-72 \% \\
5-28 \%\end{array}$ & & & \\
\hline Derrame pericárdico & $26 \%$ & & & \\
\hline Miocarditis & $53 \%$ & $39 \%$ & $50 \%$ & $73 \%$ \\
\hline Alteraciones coronarias & $8-23 \%$ & & & \\
\hline Aumento de ecogenicidad / dilatación & $17 \%$ & & & \\
\hline Aneurismas & $8-9 \%$ & $13 \%$ & $10 \%$ & $4 \%$ \\
\hline Arritmias (Extrasístoles, bloqueo) & $12 \%$ & & & \\
\hline Muerte & $2-4 \%$ & $3 \%$ & $2 \%$ & 0 \\
\hline
\end{tabular}

CV: cardiovascular. DVA: drogas vasoactivas. ECMO: asistencia ventricular extracorpórea. FEVI: fracción de eyección de ventrículo izquierdo. BNP: péptido natriurético cerebral. 


\section{Injuria Miocárdica-Miocarditis}

El concepto de injuria miocárdica se utiliza para describir a un paciente en el que al menos una medición de troponina cardíaca (cTn) sérica está por sobre del límite de referencia superior del percentil $99^{8}$. La frecuencia de la injuria miocárdica en pacientes con COVID-19 es difícil de determinar, pero es probable que su incidencia sea mayor cuando se usa cTn ultrasensible y en la medida que se efectúe su búsqueda en las presentaciones de mayor gravedad.

Existen diferentes mecanismos fisiopatológicos por los cuales probablemente se puede producir daño en el corazón. Si bien el SARS-CoV-2 puede provocar afección cardiovascular a través de un daño directo por la invasión viral a los cardiomiocitos el mecanismo principal se cree que es en forma indirecta por la respuesta inflamatoria inmune exagerada asociada a una "tormenta de citoquinas", en la cual juega un rol importante los linfocitos T Helper tipo 1 y tipo $2^{9}$. En este sentido, los hallazgos de que un alto porcentaje de los pacientes tienen serología positiva (en especial $\mathrm{IgG}$ ) en comparación con la detección del virus por RT-PCR en las series publicadas apoyan esta teoría, ya que se cree que los afectados ya habrían estado en contacto con el agente etiológico más de tres semanas antes de la admisión, lo cual es consistente con una respuesta inmunológica tardía del huésped habitual a un antígeno convencional. Otros factores fisiopatológicos derivados de este último mecanismo incluyen: el daño microvascular (donde coexisten fenómenos de angioespasmo), el aumento de la permeabilidad de los vasos y defectos de la perfusión y el estrés oxidativo e injuria por aumento de la demanda de oxígeno del miocardio secundario a la hipoxia severa.

En los pacientes que cursan con PIMS-TC se ha encontrado elevación de troponinas séricas y del péptido natriurético cerebral (BNP o NT-proBNP) sin evidencia histológica, inmunológica e inmunohistoquímica que confirmen una miocarditis de acuerdo a estos criterios. La elevación de estos biomarcadores ocurre en todas las formas clínicas siendo de mayor cuantía en presencia de shock ${ }^{10}$.

Al respecto, los pacientes graves con shock habitualmente presentan un patrón de disfunción sistólica ventricular izquierda transitoria asociado a grados variables de vasodilatación, similar a uno de tipo vasopléjico o "caliente". En forma consistente, se ha reportado mejoría en breve plazo ( 2 a 5 días) de la función miocárdica con el soporte vasoactivo y el manejo inmunomodulador o antiinflamatorio ${ }^{5,11}$, lo cual da cuenta de un mecanismo fisiopatológico más bien asociado a un "aturdimiento" y edema miocárdico por sobre un daño citopático directo con necrosis del cardiomiocito. Otros hallazgos revelan disfunción diastólica (de ventrículo izquierdo y derecho) así como derrame pericárdico leve.
Interesantemente, la elevación de las troponinas séricas es mayor a la pesquisada en la enfermedad de Kawasaki (incluso en aquellos niños con shock asociado a ésta) y a la que se ha descrito en los casos de enfermedad pulmonar severa por COVID-19 en adultos ${ }^{2,6}$. Dicha alza no se ha logrado correlacionar con los hallazgos de la Resonancia Cardíaca en estos pacientes, en los cuales se describe hiperemia y edema miocárdico, pero no realce tardío de gadolinio (sugerente de necrosis o fibrosis) como suele hallarse en las miocarditis linfocíticas virales ${ }^{12}$. Por todo esto, se ha consensuado en referirse a este patrón como una injuria miocárdica aguda no isquémica, para diferenciarla de una miocarditis propiamente $\mathrm{tal}^{13}$.

La elevación del Péptido natriurético cerebral (BNP), así como del fragmento inactivo NT-proBNP, se produce en respuesta a la sobrecarga de volumen o presión de las cámaras cardíacas pero también a la estimulación neurohumoral producido por los niveles de IL-1 y TNF- $\alpha$ presentes en estos pacientes. En la miocarditis asociada a EK su alza se asocia a disfunción diastólica y se ha utilizado como predictor de respuesta a la administración de Gamaglobulina endovenosa (IGEV), así como de aparición de aneurismas coronarios en caso de persistir sus niveles una vez administrado el fármaco mencionado. En casos de PIMS-TC su alza se ha encontrado en concordancia con el grado de disfunción ventricular e incluso su disminución puede dar una señal precoz de mejoría.

\section{Compromiso Coronario}

El compromiso coronario en PIMS-TC es relevante y probablemente, la complicación con mayor impacto a largo plazo en los afectados. Se ha reportado una frecuencia entre un 8 hasta un 33\% en las series publicadas ${ }^{14}$. Ocurre precozmente en la evolución de los pacientes (habitualmente dentro de las dos primeras semanas) y en todos los grupos de manifestaciones, incluyendo a los que sólo se manifiestan con fiebre y laboratorio de inflamación. Afecta la porción proximal de las arterias y se clasifica según el valor $\mathrm{Z}$ ecocardiográficos de acuerdo a lo establecido para el diagnóstico y seguimiento del compromiso en la $\mathrm{EK}^{15}$ como: dilatación coronaria (definida como valor $Z \geqslant 2,0$ y $<2,5$ ) aneurismas pequeños (valor $Z \geqslant 2,5 y<5$ ), medianos (valor $Z \geqslant 5 y<10$ siempre y cuando la medición absoluta sea $<8 \mathrm{~mm}$ ) y gigantes (valor $Z \geqslant 10$ o medición absoluta $\geqslant 8 \mathrm{~mm}$ ). Los seguimientos ecocardiográficos han mostrado una rápida resolución en los casos de dilataciones y en la mayoría de los aneurismas pequeños ${ }^{14}$. No se cuenta con información sobre la evolución en el tiempo de los aneurismas medianos y gigantes, y a la fecha tampoco se han publicado estudios de técnicas imagenológicas complementarias, como por ejemplo AngioTC coronario, útiles en los controles alejados de 
pacientes con EK. En ésta última enfermedad se describe también la dilatación de la raíz aórtica, lo cual debe ser considerado para su búsqueda a posterior en PIMS-TC ${ }^{16}$.

\section{Evaluación Cardiovascular Inicial}

Una vez realizado el diagnóstico de PIMS-TC según la definición que cada equipo de trabajo decida utilizar $^{2,3,4}$, se recomienda comenzar el estudio cardiovascular con electrocardiograma (ECG), troponina sérica (idealmente ultrasensible) y en los lugares donde esté disponible BNP o NT- proBNP. Con estos elementos y según los resultados, clasificar a los pacientes en dos grupos: aquéllos con PIMS-TC con compromiso cardiovascular (definido en algunas de las formas ya descritas) y el grupo PIMS-TC inflamatorio (no Kawasaki, no shock, no injuria cardiaca). Es muy importante destacar que en no pocas ocasiones pueden presentarse formas mixtas con importante superposición entre éstas e incluso los tres fenotipos de compromiso cardiovascular cambiándose de un grupo a otro y evolucionando en horas a shock y compromiso vital. En la figura 1 se describen nuestras recomendaciones de seguimiento desde el punto de vista cardiológico hospitalario, según el grupo clínico de presentación del paciente.
1. Grupo Inflamatorio (no Kawasaki, sin shock ni injuria cardiaca): Constituye un grupo de evolución clínica especial ya que los pacientes pueden evolucionar a un compromiso más grave o por el contrario, descender los valores de sus parámetros inflamatorios y su clínica en forma rápida, hasta valores normales ${ }^{14}$. En estos últimos niños, puede no efectuarse el Ecocardiograma en forma inicial pero recomendamos, dada la descripción (escasa pero presente) en la literatura de desarrollo de patología coronaria, realizar este examen entre el día $7^{\circ}-14^{\circ}$ de la enfermedad. En caso de presentarse deterioro clínico, un ascenso en los niveles de Troponina, BNP o NT-ProBNP o bien alteraciones al ECG, se deberá efectuar el seguimiento de acuerdo al fenotipo predominante como los descritos a continuación.

2. Grupo Injuria Cardíaca (Miocarditis, Pericarditis o Valvulitis): Estos pacientes requieren evaluación precoz con ecocardiograma Doppler color. En caso que éste fuera normal, se sugiere repetirlo junto al ECG entre los 7 a 14 días de enfermedad. Por el contrario, si fuese anormal, se debe efectuar una nueva evaluación a las $48 \mathrm{~h}$. Se sugiere efectuar control de biomarcadores de acuerdo a la evolución clínica cada 24-48 h hasta objetivar descenso.

3. Grupo Shock: Además del manejo intensivo atigente a este cuadro clínico, se debe efectuar eco-

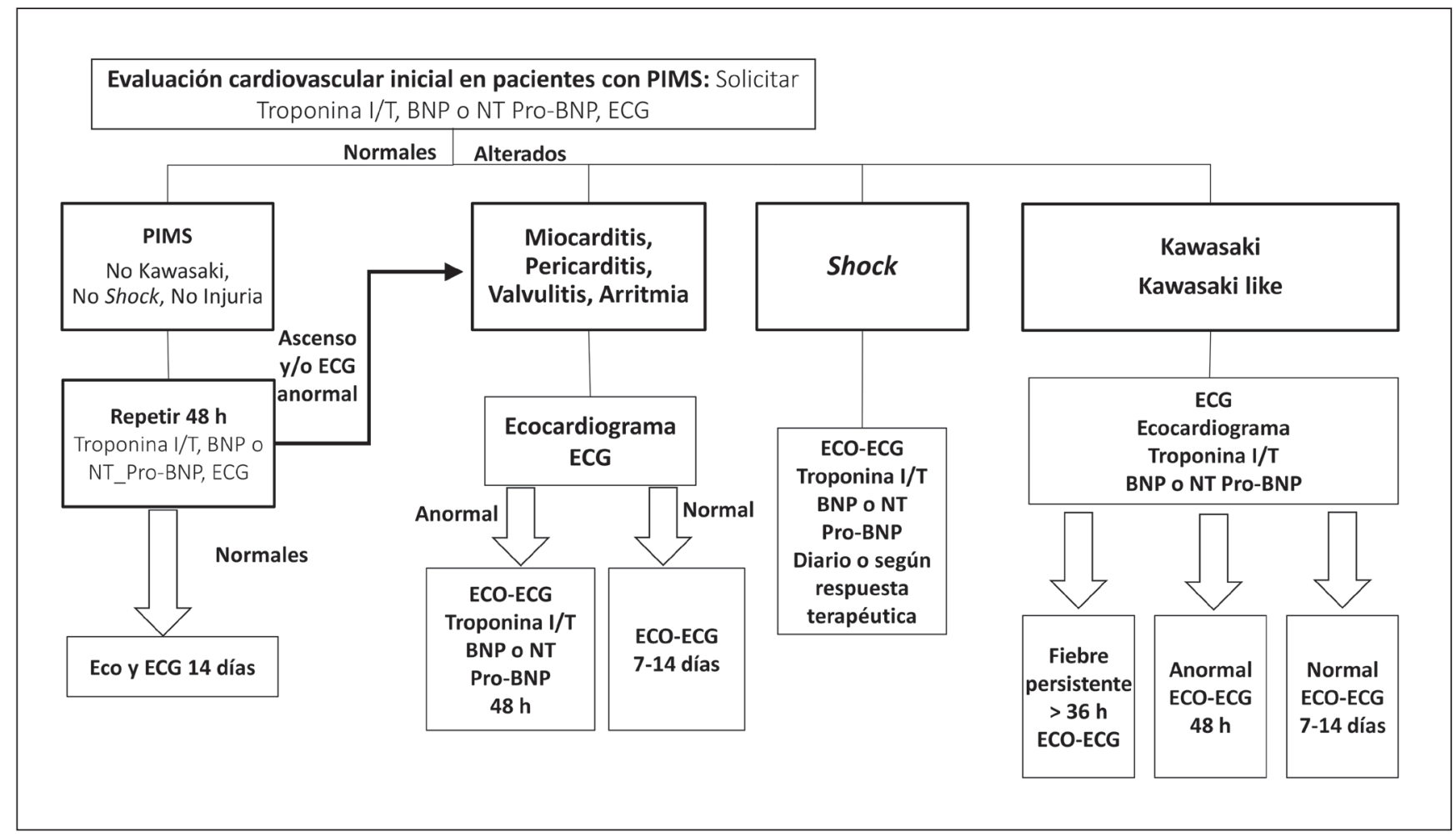

Figura 1. Recomendaciones de seguimiento cardiológico para pacientes Hospitalizados según fenotipos de PIMS-TC. PIMS-TC: Pediatric Multisystem Inflammatory Syndrome Temporally Associated with SARS-CoV-2. BNP: péptido natriurético cerebral. ECG: electrocardiograma. ECO: ecocardiografía. 
cardiograma Doppler color idealmente en forma diaria, de acuerdo al criterio del equipo tratante y la respuesta terapéutica. Complementar esta evaluación con seguimiento de curva de biomarcadores y ECG.

4. Grupo Enfermedad de Kawasaki-EK "like": En estos casos, se debe realizar ecocardiograma para descartar afectación de las arterias coronarias. Es importante destacar que la realización de éste examen no debe retrasar el inicio de la terapia si existen criterios clínicos sugerentes. Si ésta es negativo para el compromiso coronario, y el ECG y biomarcadores son normales se puede diferir el control de ecocardiograma y ECG entre los 7-14 días de la enfermedad. En caso que exista un hallazgo imagenológico anormal o bien deterioro clinico, anomalías en el ECG o elevación de biomarcadores, se recomienda un nuevo control ecocardiográfico y electrocardiográfico en $48 \mathrm{~h}$. Un tercer escenario ocurre cuando luego del tratamiento establecido por las guías del centro existe fiebre persistente. En este caso se efectuará nuevamente un Ecocardiograma Doppler color y ECG para descartar complicación y decidir terapia.

\section{Exámenes para la evaluación Cardiovascular y su utilidad}

\section{Electrocardiograma (ECG)}

Se solicita para identificar elementos que hagan sospechar miocarditis, pericarditis, isquemia y alteraciones del ritmo. Realizar en los pacientes en los cuales se sospeche PIMS-TC. Considerar su repetición cada 24-48 h en caso de: elevación o persistencia de biomarcadores altos (reflejo de inflamación aún no controlada), presencia de aneurismas coronarios o disfunción sistólica severa con fracción de eyección de ventrículo izquierdo (FEVI) $<35 \%$. Asimismo, en pacientes en unidad de cuidados intensivos con apoyo de drogas vasoactivas y/o ventilación mecánica, en caso de deterioro clínico o hemodinámico como también frente a la sospecha de refractariedad a la terapia inmunomoduladora o antiinflamatoria y previo al alta. Considerar monitorización continua o Holter del ritmo en caso de presentar arritmias y/o alteraciones de la conducción aurículoventricular (AV) o ventricular.

\section{Marcadores cardiacos (Troponina sérica I/T y BNP o Nt-ProBNP)}

Está indicada su medición en todo niño con sospecha de PIMS-TC. En caso de pesquisarse valores elevados, se debe repetir cada 24-48 h hasta objetivar descenso y luego considerar repetir cada 3-7 días hasta su normalización.

\section{Ecocardiograma Doppler color}

Todo paciente con diagnóstico de PIMS-TC debe efectuarse un ecocardiograma Doppler color en algún momento de su evolución hospitalaria, excepto en aquellos que a las $48 \mathrm{~h}$ presenten disminución de los parametros inflamatorios y tanto el ECG como los marcadores cardíacos sean normales (realizarlo a los 7-14 días de la enfermedad). Su momento de realización dependerá de la gravedad de la forma clínica de presentación. Si el paciente presenta shock y no se cuenta con la posibilidad inmediata de efectuarlo, no se debe retrasar ninguna terapia relevante en espera de la evaluación ecocardiográfica. En estos casos, consderar efectuar examen de ultrasonido cardíaco focalizado (FoCUS) por personal sanitario capacitado destinado a monitorizar cambios de conducta o terapia y/o definir soporte, evaluar función miocárdica cualitativa, presencia y cuantía de derrame pericárdico (descartar tamponamiento), estado de volemia y/o trombos intracavitarios. Esta evaluación inicial debe ser complementada posteriormente con un ecocardiograma Doppler color completo pues no reemplaza los objetivos de este.

El ecocardiograma Doppler color con técnica convencional y avanzada deberá estudiar función biventricular, presencia de disquinesia o alteraciones segmentarias, derrame pericárdico y /o compromiso valvular como también dilatación de la aorta. Además incluirá la evaluación de las arterias coronarias, utilizando los parámetros valor $\mathrm{Z}$ para precisar su tamaño de acuerdo a las recomendaciones de la Asociación Americana del Corazón para el diagnóstico, tratamiento y manejo a largo plazo de la enfermedad de Kawasaki del año $2017^{15}$ de modo de definir su compromiso como: dilatación, aneurismas pequeños, medianos o gigantes. No existe consenso hasta el momento, de qué parámetro de valor $\mathrm{Z}$ es el más fidedigno para evaluar coronarias en este contexto. Por este motivo, recomendamos usar aquel en el que más experiencia tenga el grupo hasta que exista mayor evidencia científica que apoye el uso de alguno en particular.

En la figura 2 se resumen los elementos considerados necesarios para la evaluación ecocardiográfica de estos pacientes.

\section{Tratamiento}

El tipo de terapia dependerá de la forma de presentación y debe ser siempre evaluada y decidida en conjunto con el resto de los especialistas que conforman el equipo multidisciplinario que tratará a estos pacientes. En base a lo anterior, y de acuerdo a lo establecido en la literatura existente hasta el momento, se recomienda: 
- Evaluación Funcional biventricular: con énfasis en función ventricular izquierda. Adquirir imágenes desde ejes apicales 4C, 3C y 2C, eje corto a nivel de músculos papilares. Obtener fracción de eyección de ventrículo izquierdo (FEVI) por biplano de Simpson o de Bullet (volumen de VI 5/6 área $\left(\mathrm{cm}^{2}\right)$ x longitud $(\mathrm{cm})$ ). Considerar otros métodos de evaluación de función sistólica como dP/dT mitral, S' lateral mitral, deformación miocárdica longitudinal de VI privilegiando métodos automáticos para disminuir el tiempo de análisis con el paciente (AFI, AutoStrain). Evaluación de función ventricular derecha mediante TAPSE, S' y/o cambio de área fraccional (FAC)

- Evaluación de arterias coronarias: con registro de diámetros (según guías) y medición de aneurismas según valor $Z$, de acuerdo a parámetros que se utilizen habitualmente (Sin compromiso: $<2$; dilatación aislada: 2 a $<2,5$; aneurisma pequeño: $\geq 2,5$ a $<5$; aneurisma mediano: $\geq 5$ a $<10$ y dimensión $<8 \mathrm{~mm}$; aneurisma gigante: $\geq 10,0$ dimensión $\geq 8 \mathrm{~mm}$ )

- Signos de miocardatis/pancarditis: Insuficiencias valvulares, derrame pericárdico, ecogenicidad de pericardio

- Evaluación de presencia de trombosis intracardíaca, en arterias pulmonares centrales e intracoronaria en caso de dilatación/ aneurismas.

\section{Clasificación de alteraciones:}

- Leves: Disfunción sistólica leve (FEVI 45-55\%), derrame pericárdico menos que moderado (localizado, menor $1 \mathrm{~cm}$ ), ecorefringencia o dilatación coronaria (valor Z 2 a $<2,5$ ), insuficiencias valvulares menos que moderadas

- Moderadas o severas: Disfunción sistólica con $\mathrm{FEVI}<45 \%$, derrame pericárdico circunferencial o más, aneurismas coronarios (cualquier valor $Z \geq 2,5$ ), insuficiencias valvulares moderadas o más

Figura 2. Recomendaciones de Mediciones fundamentales de Ecocardiograma Doppler color en pacientes con sospecha de PIMS-TC

1. PIMS Inflamatorio (no Kawasaki, sin shock ni injuria cardíaca): Control estricto en $48 \mathrm{~h}$ con biomarcadores inflamatorios (PCR,ferritina, fibrinógeno, Dímero-D, albúmina), hemograma y marcadores cardíacos. Si éstos van en ascenso y en ausencia de compromiso cardiovascular, se puede plantear la necesidad de corticoides a bajas dosis, en especial en presencia de: dolor abdominal, albumina menor a $3 \mathrm{~g} / \mathrm{dl}$, plaquetas menor $150.000 \mathrm{miles} / \mathrm{dL}$, linfocitos menores a 1000, Dímero D elevado 4 a 5 veces su valor normal y PCR $>50 \mathrm{mg} / \mathrm{L}$.

2. PIMS con fenotipo Kawasaki- EK "like": Dada la similitud de la forma clínica con la EK se ha preconizado el uso de inmunoglobulina endovenosa (IGEV) en dosis de 2 gramos/kilo/día y ácido acetilsalicílico (AAS) en dosis antiinflamatoria (50 $\mathrm{mg} / \mathrm{kg} / \mathrm{día}$ ), de acuerdo a las recomendaciones de la Asociación Americana del Corazón para el diagnóstico, tratamiento y manejo a largo plazo de la enfermedad de Kawasaki del año $2017^{15}$. En caso de pesquisarse uno o más aneurismas gigantes (valor $Z \geqslant 10$ o diámetro $\geqslant 8 \mathrm{~mm}$ ) se debe agregar al manejo Heparina de bajo peso molecular (HBPM). Se recomienda realizar la disminución de la dosis de AAS a rango de efecto antiplaquetario $(5 \mathrm{mg} / \mathrm{kg} /$ día), a las $48-72 \mathrm{~h}$ de paciente afebril.

La evidencia actual indica que el proceso inflamatorio desencadenado por el SARS-CoV-2 es severo e intenso, con compromiso de otros órganos que adicionan mayor gravedad y potencial mayor mortalidad a los pacientes. Dado lo anterior, se sugiere agregar el uso de corticoides a bajas dosis.
En caso de resistencia, definida como la persistencia de fiebre y de indicadores de laboratorio de inflamación después de $36 \mathrm{~h}$ de instaurada la terapia de primera línea, se puede repetir una segunda dosis de IGEV, aumentar la dosis de corticoides y, si no hay respuesta, de acuerdo a los lineamientos y la disponibilidad local, uso de infliximab o anakinra.

3. Fenotipo Shock-injuria miocárdica-miocarditis: Manejo de sostén habitual para la falla miocárdica y uso de IGEV y AAS en dosis antiinflamatoria además de corticoides a dosis intermedias. Si a pesar de lo anterior no hay respuesta, se recomienda aumentar la dosis de corticoides y considerar el uso de terapia biológica con tocilizumab o anakinra. La disminución de la dosis de AAS a antiplaquetaria, se realiza a las $48-72 \mathrm{~h}$ de permanecer el paciente afebril y se debe mantener el uso de corticoides en dosis de mantención en esquema decreciente por 3-4 semanas. Si los niños presentan riesgo de trombosis, se encuentran críticamente enfermos con pruebas de coagulación alteradas, tienen FEVI $<35 \%$ o son adolescentes se debe agregar HBPM.

Para todos los grupos clínicos se deben tener presente las siguientes precauciones:

- Previo al inicio de corticoides se debe descartar infección aguda por COVID-19 y bacteriana.

- Evitar utilizar AAS en pacientes con recuentos de plaquetas $<80.000 \mathrm{x} \mathrm{mm}^{3}$. Por el contrario, su uso se encuentra indicado en todo paciente con recuento $>450.000 \mathrm{x} \mathrm{mm}^{3}$. 
Tabla 2. Recomendaciones de Seguimiento Ambulatorio Cardiovascular en pacientes con PIMS-TC

\begin{tabular}{|c|c|c|c|c|}
\hline \multirow[t]{2}{*}{ Tiempo } & \multicolumn{2}{|c|}{ Kawasaki- Kawasaki like } & \multirow[t]{2}{*}{ Shock-Miocarditis* } & \multirow{2}{*}{$\begin{array}{l}\text { PIMS sin compromiso } \\
\text { Cardiovascular }\end{array}$} \\
\hline & Sin compromiso coronario & Con compromiso coronario & & \\
\hline 7-14 días & $E C G+E C O$ & $E C G+E C O$ & $E C G+E C O$ & $E C G+E C O$ \\
\hline $\begin{array}{l}\text { 4-6 semanas Control } \\
\text { de Troponina I/T y } \\
\text { BNP o NT-ProBNP }\end{array}$ & $\begin{array}{l}\text { ECG + ECO y recuento } \\
\text { de plaquetas. Si Recuento } \\
\text { Plaquetas normal: } \\
\text { Suspender AAS }\end{array}$ & $\begin{array}{l}\text { ECG + ECO y recuento de } \\
\text { plaquetas. } \\
\text { Mantener AAS hasta } \\
\text { resolución del compromiso } \\
\text { coronario }\end{array}$ & $\begin{array}{l}\text { ECG + ECO y recuento de } \\
\text { plaquetas. } \\
\text { Normal: control a los } 6 \text { meses } \\
\text { Anormal : mantener terapia } \\
\text { de IC y control cardiológico }\end{array}$ & \\
\hline 6 meses & $E C G+E C O$ & $E C G+E C O$ & $\begin{array}{c}\mathrm{ECG}+\mathrm{ECO}+\text { Holter de ritmo } \\
+ \text { Test de esfuerzo. } \\
\text { Normal: control al año }\end{array}$ & $E C G+E C O$ \\
\hline 1 año & $E C G+E C O$ & $E C G+E C O$ & $E C G+E C O$ & \\
\hline
\end{tabular}

- En caso de Dímero-D cuatro o cinco veces sobre el valor normal se debe utilizar HBPM como profilaxis. En caso que se pesquise o sospeche trombosis, se debe cambiar a dosis terapéutica.

- Los pacientes con trombosis documentada o con FEVI mantenida $<35 \%$ (disfunción severa) o aneurismas gigantes; deberán recibir HBPM hasta al menos dos semanas después del alta. Su mantención después de ese tiempo se decidirá según la evaluación clínica y hallazgos ecocardiográficos.

\section{Seguimiento ambulatorio}

Dado que el compromiso PIMS-TC es de reciente descripción, hasta el momento se cuenta con escasos reportes que permitan tener conocimiento del comportamiento de la enfermedad a mediano y largo plazo $^{10,14}$. Por este motivo, las recomendaciones de los diferentes grupos en su mayoría han sido elaboradas extrapolando las de EK y las de miocarditis en niños ${ }^{15,17,18}$. Teniendo lo anterior en consideración y a la espera de un mayor seguimiento en el tiempo, en los afectados de esta enfermedad se debe realizar un seguimiento estricto tanto clínico como de exámenes complementarios. En la tabla 2 se sugiere la periodicidad de controles y de exámenes complementarios de acuerdo a la presentación clínica en la fase aguda.

En términos generales, para aquellos pacientes que presentaron en la etapa aguda una FEVI $<45 \%$ o permanecen sin recuperación de la función miocárdica en el seguimiento, se debe considerar solicitar resonancia cardíaca a los 6 meses, para evaluación del miocardio, su función y definir secuelas. En segundo lugar, en aquéllos que en la etapa aguda presentan disfunción miocárdica (y/o miocarditis) moderada o severa durante el período de hospitalización, se recomienda evitar deporte competitivo y sólo permitir actividad física recreacional por seis meses. Al término de este período se debe efectuar Ecocardiograma Doppler color, ECG, Holter de arritmia y test de esfuerzo según la edad del paciente para reincorporarse a la práctica deportiva. Finalmente, todos los niños que hayan tenido compromiso cardiovascular (con y sin recuperación de éste), se recomienda realizar al año de la enfermedad ECG y ecocardiograma Doppler color.

\section{Conclusiones}

El PIMS surge como una nueva enfermedad que tiene distintos modos de presentación con el denominador común de la relación temporal con la infección por SARS-CoV2 en las semanas previas a su presentación. Ha sido reportada en niños y adolescentes de todas las razas, en su mayoría previamente sanos, dando paso a una enfermedad grave y potencialmente mortal.

La aparición de compromiso cardiovascular secundario a PIMS-TC es uno de los elementos que ha alertado a clínicos a tener un alto grado de sospecha de la enfermedad. Lo anterior se ve refrendado en la observación que la afección cardíaca y coronaria puede presentarse independiente de la forma clínica y aún en pacientes no graves. El poco tiempo transcurrido desde su descripción (fines de abril de este año a la fecha), y por lo tanto la ausencia de segiuimiento hacen que el manejo en la fase aguda y posteriomente la búsqueda de secuelas deba ser enfrentado de una forma sistematizada y estricta.

Las decisiones frente a cada caso clínico en parti- 
Síndrome inflamatorio multisistémico - P. Álvarez Z. et al

cular deben ser abordadas de forma multidisciplinaria y orientada a la particularidad que presente cada paciente.

\section{Conflicto de intereses}

Los autores declaran no tener conflicto de intereses.

\section{Referencias}

1. Belot A, Antona D, Renolleau S, et al. SARS-CoV-2-related paediatric inflammatory multisystem syndrome, an epidemiological study, France, 1 March to 17 May 2020. Euro Surveill. 2020;25(22):2001010. doi:10.2807/15607917.ES.2020.25.22.2001010.

2. Royal College of Paediatrics and Child Health, editor. Guidance: Paediatric multisystem inflammatory syndrome temporally associated with COVID-19. UK: Royal College of Paediatrics and Child Health; 2020.

3. Center of Control Disease. Multisystem Inflammatory Syndrome in Children (MIS-C) Associated with Coronavirus Disease 2019 (COVID-19). Official Health Advisory. [Internet]. [citado el 25 de julio de 2020]. Disponible en: https:// emergency.cdc.gov/han/2020/han00432. asp.

4. World Health Organization. Multisystem inflammatory syndrome in children and adolescents with COVID-19. Published May 15, 2020. Accessed May 22, 2020. https://www.who. int/publications-detail/ multisystem-inflammatorysyndrome- inchildren-and-adolescents-withcovid-19.

5. Feldstein LR, Rose EB, Horwitz SM, et al. Multisystem inflammatory syndrome in U.S. children and adolescents. $\mathrm{N}$ Engl J Med. DOI: 10.1056/NEJMoa2021680.

6. Toubiana J, Poirault C, Corsia A, Bajolle F, Fourgeaud J et al. Outbreak of Kawasaki disease in children during COVID-19 pandemic: a prospective observational study in Paris, France. medRxiv. Preprint posted May 2020. doi: 10.1101/2020.05.10.20097394.

7. Whittaker E, Bamford A, Kenny J, et al. Clinical characteristics of 58 children with a pediatric inflammatory multisystem syndrome temporally associated with SARS-CoV-2. JAMA 2020 June 8 (Epub ahead of print).

8. Thygesen K, Alpert JS, Jaffe AS, et al; Executive Group on behalf of the Joint
European Society of Cardiology (ESC)/ American College of Cardiology (ACC)/ American Heart Association (AHA)/ World Heart Federation (WHF) Task Force for the Universal Definition of Myocardial Infarction. Fourth Universal Definition of Miocardial Infarction. Circulation. 2018;138(20):e618-e651. doi: 10.1161/CIR.0000000000000617.

9. Mehta P, McAuley DF, Brown M, Sanchez E, Tattersall RS, Manson JJ; HLH Across Speciality Collaboration, UK. COVID-19: consider cytokine storm syndromes and immunosuppression. Lancet. 2020;395:1033-1034

10. Ramcharan T, Nolan O, Lai CY, et al. Paediatric inflammatory multisystem syndrome: temporally associated with SARS-CoV-2 (PIMS-TS): cardiac features, management and short-term outcomes at a UK tertiary paediatric hospital. Pediatr Cardiol. 2020. https://doi.org/10.1007/ s00246-020-02391.

11. Belhadjer Z, Méot M, Bajolle F, et al. Acute heart failure in multisystem inflammatory syndrome in children (MIS-C) in the context of global SARSCoV-2 pandemic. Circulation 2020 May 17

12. Blondiaux E, Parisot $\mathrm{P}$, Redheuil A, et al. Cardiac MRI of Children with Multisystem Inflammatory Syndrome (MIS-C) Associated with COVID-19: Case Series. Radiology 2020. https://doi. org/10.1148/radiol.2020202288

13. Sandoval Y, Januzzi JL Jr, Jale AS. Cardiac Troponin for the Diagnosis and RiskStratification of Myocardial Injury in COVID-19: JACC Review Topic of the Week. J Am Coll Cardiol 2020;Jul 3.

14. Jhaveri S, Ahluwalia N, Kaushik S, Trachtman R, Kowalsky S, Aydin S, Stern K. Longitudinal Echocardiographic Assessment of Coronary Arteries and Left Ventricular Function Following Multisystem Inflammatory Syndrome in Children (MIS-C). The Journal of Pediatrics 2020. https://doi.org/10.1016/j. jpeds.2020.08.002.
15. McCrindle BW, Rowley AH, Newburger JW, et al. Diagnosis, treatment, and long-term management of Kawasaki disease: a scientific statement for health professionals from the American Heart Association. Circulation. 2017;135(17):e927-99. doi: 10.1161/ CIR.0000000000000484. On behalf of the American Heart Association Rheumatic Fever, Endocarditis, and Kawasaki Disease Committee of the Council on Cardiovascular Disease in the Young; Council on Cardiovascular and Stroke Nursing; Council on Cardiovascular Surgery and Anesthesia; and Council on Epidemiology and Prevention.

16. Riphagen S, Gómez X, GonzálezMartínez C, Wilkinson N, Theocharis P. Hyperinflammatory shock in children during COVID-19 pandemic. Lancet. 2020;395(10237):1607-8. doi: 10.1016/ S0140- 6736(20)31094-1.

17. Bohn D. Acute viral myocarditis in children: guidelines. Pediatr Crit Care Med. 2006;S1-S24.

18. Kindermann I, Barth C, Mahfoud F, et al. Update on myocarditis. J Am Coll Cardiol. 2012;59:779-92.

19. Dufort E, Koumans E, Chow E, et al. Multisystem Inflammatory Syndrome in Children in New York State. N Engl J Med [Internet]. 2020; Available from: http://www.ncbi.nlm.nih.gov/ pubmed/32598830.

20. Cofré F, Izquierdo G, Tapia L, Poli C, Delpiano L, Conca N. Recomendación para la sospecha diagnóstica y manejo inicial del Síndrome Inflamatorio Multisistémico (SIM-COVID-19) en contexto pandemia SARS-CoV-2. Rev Chil Infectología. 2020;(1):1-9.

21 Davies P, Evans C, Kanthimathinathan H, et al. Intensive care admissions of children with paediatric inflammatory multisystem syndrome temporally associated with SARS-CoV-2 ( PIMS-TS ) in the UK : a multicentre observational study. Lancet. 2020;2(20):1-9. 